\title{
Quantification of EEG Brain Activity in the Self-Paced Regime
}

\author{
Joana Silva ${ }^{1}$, A. Martins da Silva ${ }^{2}$, Luís Coelho ${ }^{3}$ \\ ${ }^{1}$ Department of Electrical and Computer Engineering, Faculty of Engineering University of \\ Porto, Porto, Portugal (up201708861@fe.up.pt); ${ }^{2}$ Neurophysiology Service, Hospital Santo \\ António/CH Porto and UMIB-ICBAS, Universidade do Porto, Porto, Portugal \\ (mas@icbas.up.pt); ${ }^{3}$ Physics Department, Instituto Superior de Engenharia do Porto, Porto, \\ Portugal (Ifc@isep.ipp.pt) and INEGI- Laboratory of Optics and Experimental Mechanics
}

\begin{abstract}
The processing of motor, sensory and cognitive information by the brain can result in changes of the electroencephalogram (EEG) by Event Related Desynchronization (ERD) or Event Related Synchronization (ERS). The first one concerns a decrease in the amplitude of a rhythmic activity while the second corresponds to its increase. The analysis of these two phenomena in specific frequency bands - alpha $(8-13 \mathrm{~Hz})$ and beta (14-30 Hz) - allows the understanding of the cerebral activity. This study focuses on the quantification of cerebral activity by determining the ERD and ERS on the referred band, induced by self-paced movements, by using EEGLAB and MATLAB tools. This was achieved by the creation of a new and automatic quantification algorithm. The results indicate that a greater desynchronization of the signal is accompanied by a decrease in the amplitude of the same. As a conclusion, the cerebral activity varies in terms of synchronization and desynchronization among certain frequency bands in several zones, according to the tasks performed.
\end{abstract}

Keywords: Electroencephalogram (EEG), Event Related Desynchronization (ERD), Event Related Synchronization (ERD), Alfa Band, Beta Band, Self-Pacing

Type: Research Article

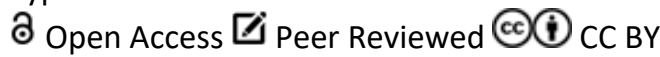

\section{Introduction}

The electroencephalogram records the amplified electrical activity coming from the brain's nerve cells - the neurons. Through the placement of electrodes on the scalp, it is possible to obtain a sinusoidal signal, which is generated by the electrical fields that are the basis of the origination of the EEG (Wave Morphology 2017).

It is known that the characteristics of the waves recorded in the EEG change according to the physiological condition of the individual and they are different from waking, to sleeping and to dreaming, with changes at amplitude and frequency. The EEG common frequencies bands are alpha $(8-13 \mathrm{~Hz})$, beta $(14-30 \mathrm{~Hz})$, theta $(4-7,5 \mathrm{~Hz})$ and delta $(0.1-3,5 \mathrm{~Hz})$ (Kitahara et al. 2017). These frequency bands are more evident in specific brain zones, at rest or depending on the stimuli to which the brain is subjected. These stimuli / events produce responses of different neuronal structures that alter EEG dynamics. Such changes can be classified as Event Related Desynchronization (ERD) and Event Related Synchronization (ERS) (Pfurtscheller 2001). The first relates to a decrease in the amplitude of a rhythmic activity, when there is an increase in excitability against cortical activation, while the second one corresponds to an increase in the amplitude of a rhythmic activity, resulting from a deactivation of the same cortical area (Da Silva and Niedermeyer 2010; Pfurtscheller 2001). 
According to the previous explanation, there are three different states of cortical processing (Da Silva and Niedermeyer 2010):

1. Neutral or resting state, with no specific processing of sensory, motor or cognitive information;

2. Active state, with high information processing in a specific system and increased excitability of cortical neurons. This state is characterized by ERD;

3. Deactivated state, with reduced information processing in a specific system and decreased cortical excitability. This state is characterized by ERS in the lower alpha and beta bands.

Since ERD/ERS is a response of different neuronal structures, the objective of this study is to quantify this response within specific frequency bands - alpha and beta - in individuals when placed in self-pacing activity. This term refers to a self-managed activity, whose stimuli are triggered by the individual, in a rhythm of their own and do not originate from the outside (for example, counting every 5 seconds and, at the end of each time interval, lifting a finger) (Vilhena 2003). One of the aims of this type of study is, for example, to decode voluntary movement before the action towards implementing practical brain-machine interface (BMI) technology for intuitive neuroprosthesis (Lew et al. 2012). Other example is the study made by Lana, Adorno and Tierra-criollo (2013), where it was analyzed the relation between the movement of an anthropomorphic robotic manipulator and the brain cortex response. More recently, Qiu and his team studied the comparison between ERD patterns during active movement, passive movement and functional electrical stimulation (FES)-induced movement of the lower limb, concluding that the quantification of ERD patterns could be expected as a potential technique to evaluate brain response during FES-induced movement (Qiu et al. 2016).

For ERD/ERS quantification, tests were performed in 3 different participants. The purpose was to accomplish a study of the diverse values obtained and to define if they can be used as an individual measure of the normality of the cerebral activity, analysing the variations of the signal in terms of locations of the scalp.

\section{Materials and Methods}

Three healthy individuals, two females, one male, with similar age (young adults - 20-22 years old), highly educated, and not using pharmacological treatments, were studied on a voluntary base. For the acquisition of the EEG signals we studied all individuals, placing electrodes on the scalp surface according to the 10-20 International System (Da Silva and Niedermeyer 2010). In this study, only 16 electrodes were placed in the right and left hemispheres, as shown in Figure 1 a) and b). This number of electrodes was sufficient to capture information from the various zones of the scalp and to reduce the interference between the signals of neighbouring channels. Since the electric potential is a relative magnitude, it was necessary to use a reference, given by the sum of the electrodes electrical signals acquired in each channel and divided by the total number of channels (average). In addition to the electrodes placed on the scalp, another three were placed: one in the extensor/ flexor of the right hand, one in the left-hand extensor/ flexor and another on the chest to record the ECG. 


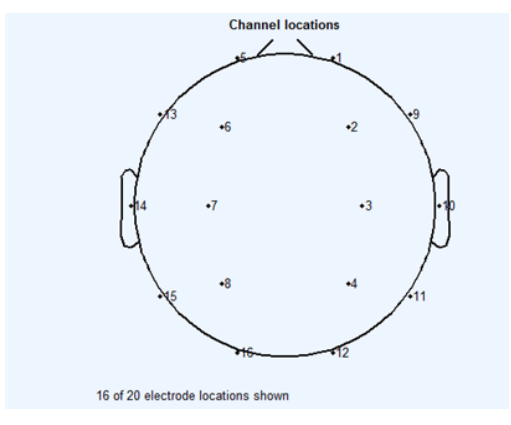

a)

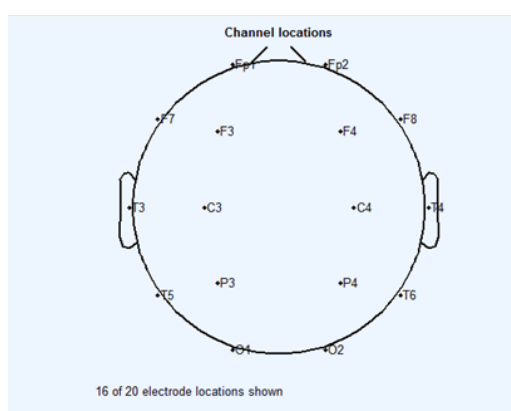

b)

Figure 1: Positioning of the electrodes extracted from EEGLAB loaded data:

a) by index; b) by name

The experimental procedure comprises two parts: one at rest and the other of self-pacing. First, the signal was recorded with the eyes open for two minutes, followed by acquisition with eyes closed for two minutes. After, we ask the individual to open the eyes and close three times to see the artefacts. In the second part, the participant, in waking state, counts for five seconds, then rises up his/her right hand. This was repeated for up to five minutes. When finished, the individual repeats the same procedure for the left hand.

The acquisition of the signals was done through the Brain Wave II EEG equipment, that works with BWAnalysis software (Neurovirtual 2017). This software, running in computer with Windows operating system and using a $300 \mathrm{~Hz}$ sampling frequency, exports the data to edf. files that, posteriorly, are loaded and worked in EEGLAB (Delorme and Makeig 2004; SCCN 2017) and MATLAB (MathWorks 2017). With the signals loaded, the software EEGLAB provided graphic analysis while MATLAB provided the obtained of numerical data.

The ERD calculation, in MATLAB, followed the classical method, which are explained in the next steps (Kitahara et al. 2017; Kumar and Bhuvaneswari 2012):

1. Filter the signal with a bandpass referring to the frequency to be studied;

2. Raise the signal squared to get its energy;

3. Average the amplitudes for each sample in all the tests;

4. Subtract for each sample the mean of the sum of all amplitudes of the signal;

5. Add the amplitudes of all samples, within each test, and divide by the total number of samples;

6. Calculate the ERD, which is given by: $E R D \%=((A-R) / R) \times 100$, where $A$ is the calculated power in the analysis interval and $R$ is the bandwidth in all assays.

\section{Results and Discussion}

In MATLAB, the signal from each electrode was filtered according to the frequency band of interest and the mean amplitudes of each were calculated through the procedure previously explained. These amplitudes were then compared with the basal activity (at rest) of the participant, in order to observe their evolution before a situation of brain activity. Graphics 1 and 2 illustrate the comparison between the amplitude of each channel at rest and the amplitude of each active channel, in alpha band and in beta band, respectively, for the participant 1. 


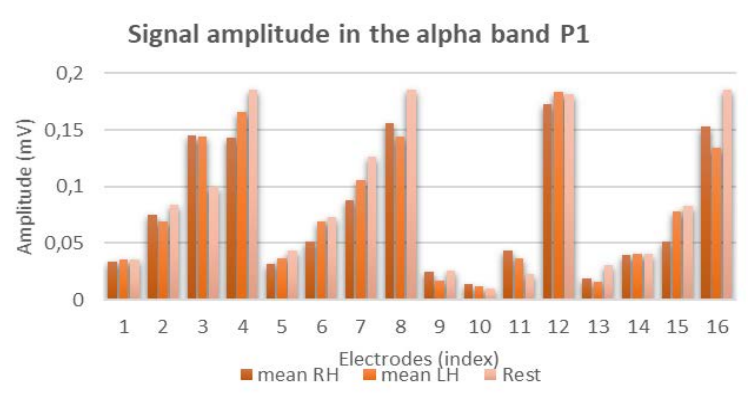

Graphic 1: Comparison between the amplitudes at rest and during activity in the right hand (RH) and left (LH) movement situations, in the alpha frequency band, for the participant 1 (P1)

Signal amplitude in the beta band P1

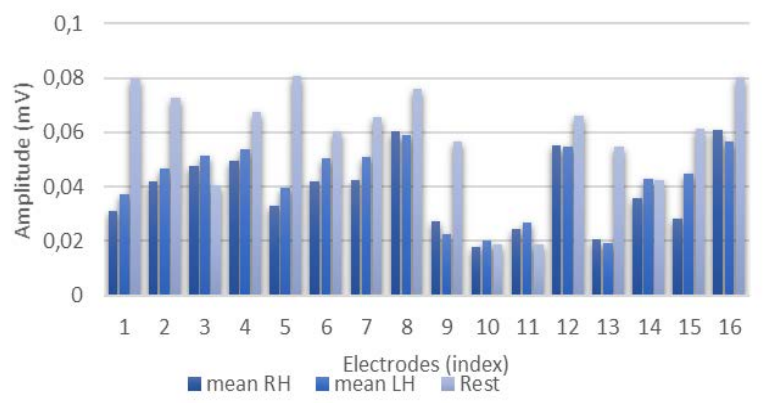

Graphic 2: Comparison between the amplitudes at rest and during activity in the right hand (RH) and left (LH) movement situation, in the beta frequency band, for the participant 1 (P1)

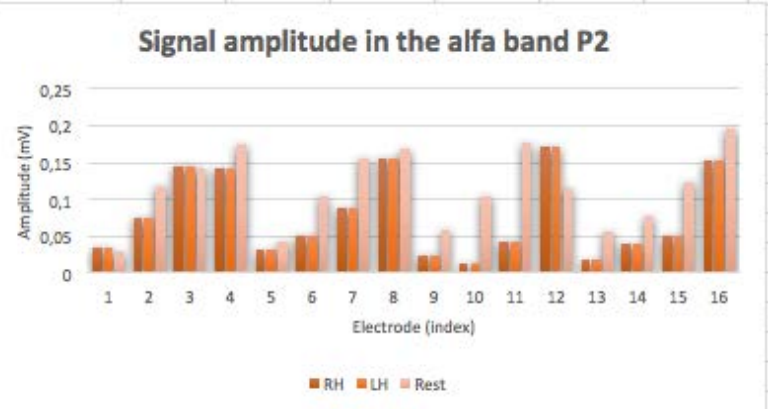

Graphic 3: Comparison between the amplitudes at rest and during activity in the right hand (RH) and left (LH) movement situation, in the alpha frequency band, for the participant 2 (P2)

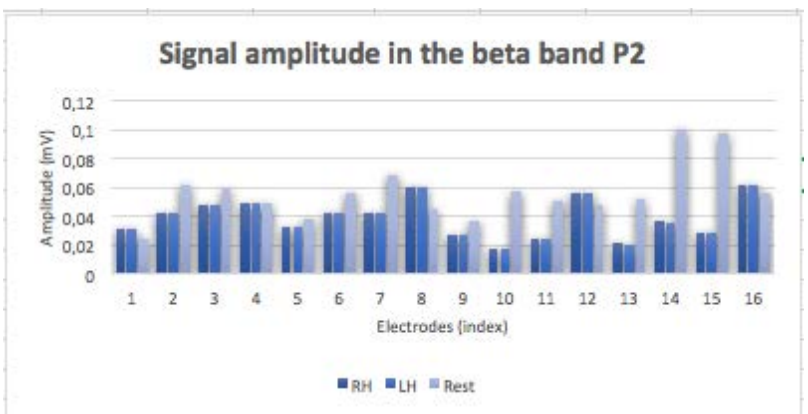

Graphic 4: Comparison between the amplitudes at rest and during activity in the right hand (RH) and left (LH) movement situation, in the beta frequency band, for the participant 2 (P2) 


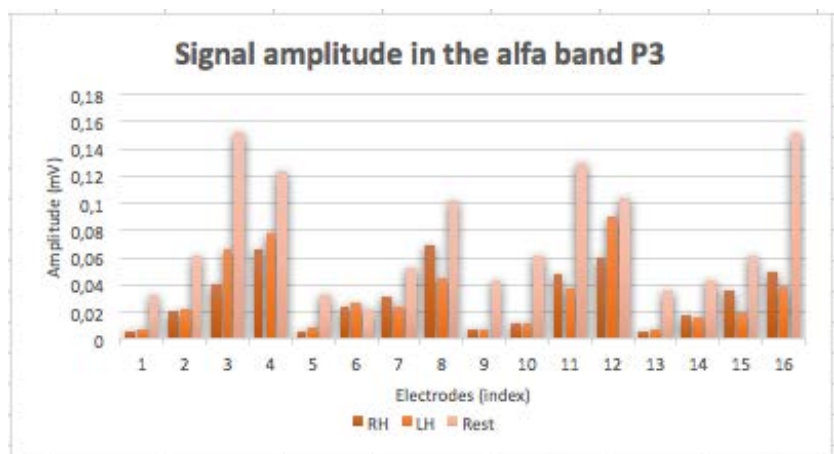

Graphic 5: Comparison between the amplitudes at rest and during activity in the right hand (RH) and left (LH) movement situation, in the alpha frequency band, for the participant 3 (P3)

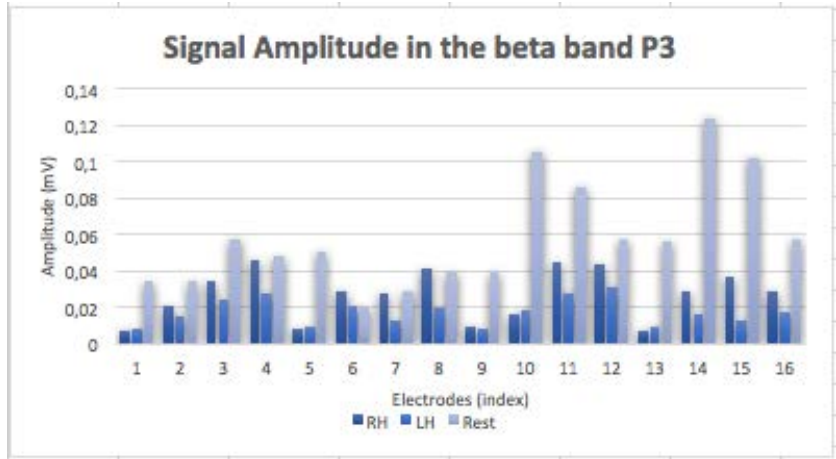

Graphic 6: Comparison between the amplitudes at rest and during activity in the right hand (RH) and left (LH) movement situation, in the beta frequency band, for the participant 3 (P3)

According to the previous graphs, it is observed that the amplitude of rest is greater in almost all participants comparing to the amplitudes recorded in situations of activity. These data indicate that a desynchronization activity is accompanied by a decrease in signal amplitude, as explained in the introduction (Kumar and Bhuvaneswari 2012; Lew et al. 2012). Although the trend observed in the charts is in accordance with the literature, there are some channels that register an amplitude increase. This relates to the fact that, in different locations, in real time, a synchronization is accompanied by an increase in amplitude and indicates a reduction without information processing.

The amplitudes observed previously suggest that, in fact, during the activities a signal synchronization of the electrodes occurred. In order to quantify this activity (ERD), calculations were performed in MATLAB not only with the purpose of recording the desynchronization values, but also with the objective of comparing them in the different frequency bands.

Regarding the frequency bands, as we have seen, it is known that the alpha band desynchronization is more evident in the posterior zones and in the beta band, in the lateral and anterior zones of the scalp. However, according to the results of Table 1, it is possible to observe that the higher values of desynchronization (highlighted in black), correspond to anterior zones of the scalp, so it was expected that these appeared in the posterior part. It can be explained by the role of such zones on the processing of the mental activity. In Table 2 , the highlighted values correspond to the desynchronization of the signals of the electrodes positioned on the lateral and anterior parts of the scalp, which is in conformity with the expected one. However, the participant 3 (P3) was the one that showed a better result, and the higher percentages appear in electrodes in the posterior zone, which is characterized by the appearance of frequencies of the beta band. 
After the analysis of the desynchronization values, it is also important to know what happens before and after the stimulus, in terms of signal power and amplitude. For this study, the signs were cut in the EEGLAB and spectral maps were elaborated for each participant, however, only the results of the participant 1 will be exposed here, due to the extension of data.

\begin{tabular}{c|ccc} 
Index & ERD P1 (\%) & ERD P2 (\%) & ERD P3 (\%) \\
\hline $\mathbf{1}$ & $-99,59$ & $-99,58$ & $-99,08$ \\
$\mathbf{2}$ & $-98,02$ & $-98,01$ & $-99,81$ \\
$\mathbf{3}$ & $-92,71$ & $-92,98$ & $-98,85$ \\
$\mathbf{4}$ & $-93,48$ & $-93,21$ & $-95,58$ \\
$\mathbf{5}$ & $-99,60$ & $-99,61$ & $-99,99$ \\
$\mathbf{6}$ & $-99,14$ & $-99,09$ & $-99,76$ \\
$\mathbf{7}$ & $-97,50$ & $-97,45$ & $-99,20$ \\
$\mathbf{8}$ & $-92,34$ & $-91,76$ & $-93,90$ \\
$\mathbf{9}$ & $-99,79$ & $-99,79$ & $-99,98$ \\
$\mathbf{1 0}$ & $-99,93$ & $-99,93$ & $-99,94$ \\
$\mathbf{1 1}$ & $-99,33$ & $-99,32$ & $-97,63$ \\
$\mathbf{1 2}$ & $-90,38$ & $-90,54$ & $-96,94$ \\
$\mathbf{1 3}$ & $-99,86$ & $-99,87$ & $-99,99$ \\
$\mathbf{1 4}$ & $-99,47$ & $-99,41$ & $-99,90$ \\
$\mathbf{1 5}$ & $-99,09$ & $-99,07$ & $-99,01$ \\
$\mathbf{1 6}$ & $-92,39$ & $-91,80$ & $-96,44$
\end{tabular}

Table 1: Desynchronization (in \%) associated with each electrode, in the right-hand movement situation, analysed in the alpha frequency band, for the three participants (P1, P2 and P3)

\begin{tabular}{c|ccc} 
Index & ERD P1 (\%) & ERD P2 (\%) & ERD P3 (\%) \\
\hline $\mathbf{1}$ & $-99,68$ & $-99,6191$ & $-99,983$ \\
$\mathbf{2}$ & $-99,42$ & $-99,40$ & $-99,84$ \\
$\mathbf{3}$ & $-99,21$ & $-99,22$ & $-99,57$ \\
$\mathbf{4}$ & $-99,18$ & $-99,18$ & $-99,23$ \\
$\mathbf{5}$ & $-99,63$ & $-99,60$ & $-99,97$ \\
$\mathbf{6}$ & $-99,34$ & $-99,37$ & $-99,69$ \\
$\mathbf{7}$ & $-99,36$ & $-99,3$ & $-99,72$ \\
$\mathbf{8}$ & $-98,86$ & $-98,82$ & $-99,35$ \\
$\mathbf{9}$ & $-99,60$ & $-99,61$ & $-99,98$ \\
$\mathbf{1 0}$ & $-99,50$ & $-99,58$ & $-99,89$ \\
$\mathbf{1 1}$ & $-99,73$ & $-99,72$ & $-99,25$ \\
$\mathbf{1 2}$ & $-98,10$ & $-98,99$ & $-99,33$ \\
$\mathbf{1 3}$ & $-99,75$ & $-99,73$ & $-99,98$ \\
$\mathbf{1 4}$ & $-99,48$ & $-99,48$ & $-99,53$ \\
$\mathbf{1 5}$ & $-99,72$ & $-99,70$ & $-99,26$ \\
$\mathbf{1 6}$ & $-98,81$ & $-98,80$ & $-99,68$
\end{tabular}

Table 2: Desynchronization (in \%) associated with each electrode, in the right-hand movement situation, analysed in the beta frequency band, for the three participants (P1, P2 and P3) 


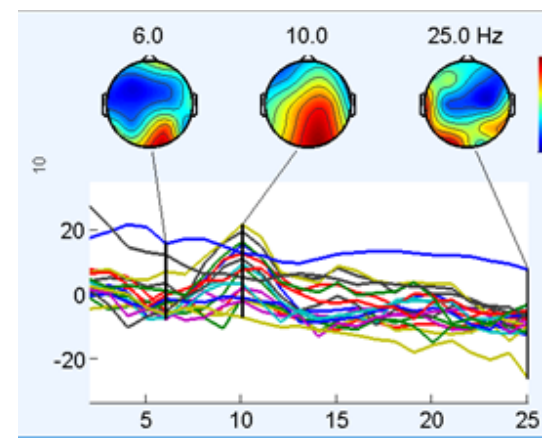

a)

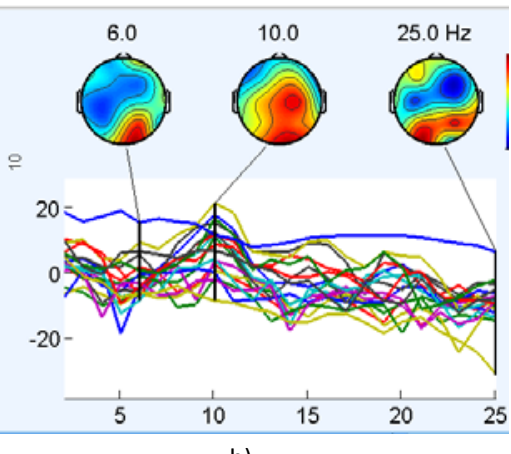

b)

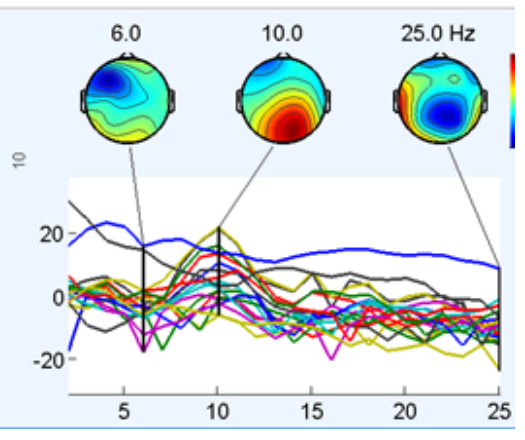

c)

Figure 2: Spectral maps related to the activity resulting from a stimulus with the right hand, for the participant 1: a) in the total time of the stimulus; b) before the stimulus; b) after the stimulus
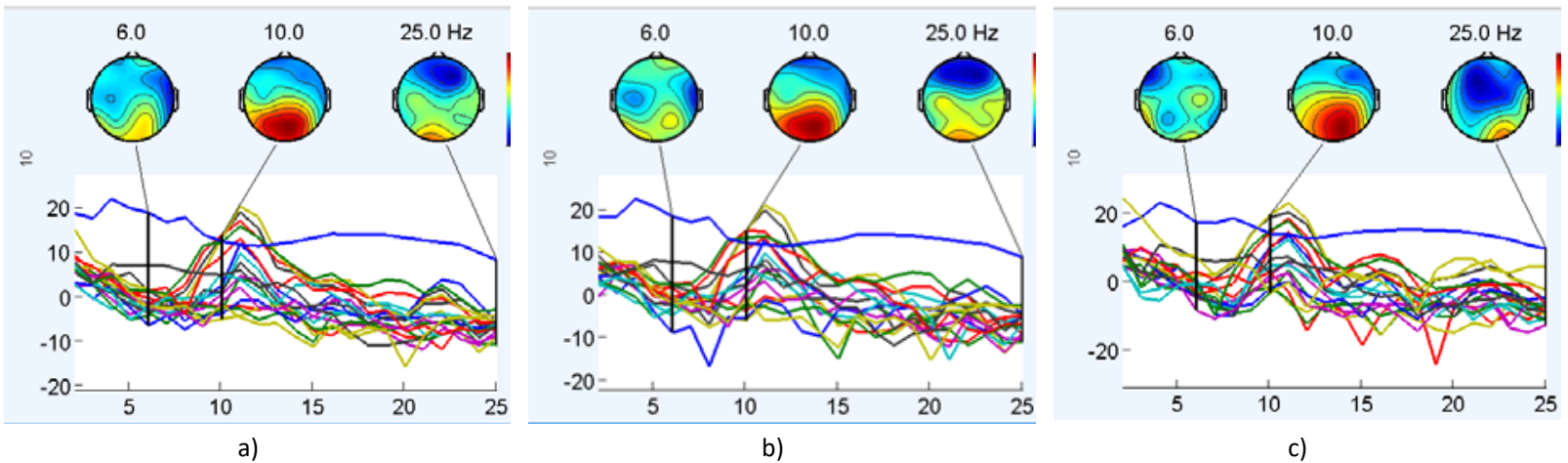

Figure 3: Spectral map related to the activity of a left-hand stimulus, for the participant 1: a) in the total time of the stimulus; b) before the stimulus; $c$ ) after the stimulus

Figures 2 and 3 show that alpha band frequencies are more prominent in the posterior region of the scalp along the right and left-hand movement action. This is seen by the orange color on these regions.

In Figure $2 \mathrm{c}$ ), it is possible to emphasize that the greater spectral power of the scalp tends more towards the left hemisphere, which is related to an activity of the right-hand. On the other hand, in Figure $3 \mathrm{c}$ ), it is observed that the power was more evident in the right side, which is related to an activity of the left-hand.

In both left and right-hand movement, the spectra indicate that before the stimulus, there is already a desynchronization in the posterior zone of the scalp, which means the preparation of the brain, by electrical responses, for doing a task. After the stimulus, the activity is much reduced.

\section{Conclusions}

In this study, all the methods of processing EEG signals were performed with the purpose of quantifying the brain responses to stimuli, by the development of an algorithm. According to the literature used, it is known that the response of brain activity changes depending on the type of stimulus to which the brain is submitted and depending on the brain areas, therefore, the calculation of the signal varies according to a task. The results obtained are in conformity with this assertion because, for the movement situation of the right hand and the left hand, the brain activations, in terms of power, frequency and amplitude, have changed.

The results showed that the amplitude of the resting signal was greater than that one registered during the execution of a task, when the amplitude decreases. This was related to an increase in the desynchronization. For future work and for obtaining more reliable 
results, it would be important to analyze the signs of more information, with a larger sample, making the elaborated algorithm to be tested in more cases, increasing its feasibility and allowing its improvement.

\section{References}

Da Silva, F. H. L., and E. Niedermeyer. 2010. Electroencephalography: Basic principles, clinical applications, and related fields. Philadelphia: Lippincott Williams \& Wilkins.

Delorme, Arnaud, and Scott Makeig. 2004. "EEGLAB: An open source toolbox for analysis of single-trial EEG dynamics including independent component analysis". Journal of Neuroscience Methods 134 (1): 9-21. DOI: 10.1016/j.jneumeth.2003.10.009.

Kitahara, K., Y. Hayashi, S. Yano, and T. Kondo. 2017. "Target-directed motor imagery of the lower limb enhances event-related desynchronization". PLOS ONE 12 (9). DOI: 10.1371/journal.pone.0184245.

Kumar, J. Satheesh, and P. Bhuvaneswari. 2012. "Analysis of electroencephalography (EEG) signals and its categorization - A study". Procedia Engineering 38: 2525-36. DOI: 10.1016/j.proeng.2012.06.298.

Lana, Ernesto Pablo, Bruno Vilhena Adorno, and Carlos Julio Tierra-criollo. 2013. "An ERD/ERS analysis of the relation between human arm and robot manipulator movements". Paper presented at 2013 ISSNIP Biosignals and Biorobotics Conference: Biosignals and Robotics for Better and Safer Living (BRC). 18-20 Feb. 2013. DOI: 10.1109/BRC.2013.6487461.

Lew, E., R. Chavarriaga, H. Zhang, M. Seeck, and J. d. R. Millán. 2012. "Self-paced movement intention detection from human brain signals: Invasive and non-invasive EEG". Paper presented at 2012 Annual International Conference of the IEEE Engineering in Medicine and Biology Society. Aug. 28 2012-Sept. 1 2012. DOI: 10.1109/EMBC.2012.6346665.

MathWorks. 2017. "MathWorks". Accessed November 18, 2017. https://www.mathworks.com/products/matlab.html.

Neurovirtual. 2017. "BWAnalysis EEG/ICU/LTM software". Accessed November 18, 2017. http://neurovirtual.com/equipment/software-bwanalysis/.

Pfurtscheller, G. 2001. "Functional brain imaging based on ERD/ERS". Vision Research 41 (10): 1257-60. DOI: 10.1016/S0042-6989(00)00235-2.

Qiu, S., W. Yi, J. Xu, H. Qi, J. Du, C. Wang, F. He, and D. Ming. 2016. "Event-related beta EEG changes during active, passive movement and functional electrical stimulation of the lower limb". IEEE Transactions on Neural Systems and Rehabilitation Engineering 24 (2): 283-90. DOI: 10.1109/TNSRE.2015.2476481.

SCCN. 2017. "What is EEGLAB?". Accessed November 18, 2017. https://sccn.ucsd.edu/eeglab/index.php.

Vilhena, Estela Maria dos Santos Ramos. 2003. "Análise quantitativa dos potenciais cerebrais ERP300 em adultos jovens". Master's thesis, Faculdade de Engenharia, Universidade do Porto. http://catalogo.up.pt/F/?func=direct\&doc_number=000469929.

Wave Morphology. 2017. "Electroencephalographic artifacts". Accessed November 18, 2017. DOI: B978-0-7506-7478-2/00015-2. 